\title{
Relaciones filogenéticas entre telmatobiinidos (Anura, Ceratophryidae, Telmatobiinae) de los Andes centrales basado en la morfología de los estados larval y adultos
}

\author{
Phylogenetic relationships between telmatobiinids (Anura, Ceratophryidae, \\ Telmatobiinae) of central Andes based on morphology of larval and adult \\ stages \\ César Aguilar y Niels Valencia
}

Museo de Historia Natural, Universidad Nacional Mayor de San Marcos, Av. Aron Av. Arenarta 14-0434, Lima 14, Perú. Email César Aguilar:caguilarp@ unmsm.edu.pe

Presentado: 23/02/2009 Aceptado: 10/04/2009 Publicado online: 28/08/2009

\begin{abstract}
Resumen
Batrachophrynus y Telmatobius son los dos únicos géneros reconocidos de Telmatobiinae presentes en los Andes centrales. Las especies de ambos géneros presentan adaptaciones para la vida en la altitud de los Andes siendo de hábitos acuáticos o semiacuáticos en bofedales, riachuelos, lagunas o lagos altoandinos. Este estudio presenta las relaciones filogenéticas entre Batrachophrynus y 13 especies de Telmatobius utilizando caracteres morfológicos larvales y adultos, incluyendo caracteres diagnósticos para Batrachophrynus y Telmatobius, y las sinapomorfías sugeridas para Telmatobius. El análisis filogenético dio como resultado 20 árboles igualmente parsimoniosos con una longitud de 56 pasos. Batrachophrynus forma un grupo monofilético anidado dentro del clado de Telmatobius. En este estudio, la mayoría de sinapomorfías que sustentan a Telmatobius (incluyendo a Batrachophrynus) provienen de la morfología larval y estas sinapomorfías probablemente soporten a todo el género
\end{abstract}

Palabras clave: Batrachophrynus, Telmatobius, Morfología, Filogenia, Andes centrales.

\section{Abstract}

Batrachophrynus and Telmatobius are the two genus of Telmatobiinae from the central Andes. Both genera have species with adaptations for life at high altitude in the Andes, with aquatic or semi-aquatic habits in creeks, lagoons and lakes. The objective of this study is to evaluate the phylogenetic relationships between Batrachophrynus and 13 species of Telmatobius from the central Andes using larval and adult morphology including diagnostic characters for Batrachophrynus and Telmatobius, and putative sinapomorphies for Telmatobius. The phylogenetic analysis showed 20 parsimonious trees with 56 steps length. The results of this study hypothesize that the species assigned to Batrachophrynus form a monophyletic group nested within Telmatobius. In this study, most of the synapomorphies that support Telmatobius (including Batrachophrynus) come from larval morphology and these sinapomorphies will probably support the whole genus.

Keywords: Batrachophrynus, Telmatobius, Morphology, phylogeny, central Andes

\section{Introducción}

Los géneros Batrachophrynus y Telmatobius, comprenden un grupo de ranas endémicas de las partes altas de los Andes, desde los $1500 \mathrm{~m}$ hasta más de $5000 \mathrm{~m}$, con adaptaciones a la vida acuática o semiacuática (Vellard 1951, 1952; De Macedo 1950, 1976; Trueb 1979; Czopeck 1983; Cei 1986; Seimon et al. 2007). Telmatobius es conocido con 58 especies y habita los Andes por el norte desde Ecuador hasta Argentina y Chile por el sur (Barrionuevo y Baldo 2009, Frost 2009). La mayoría de las especies distribuidas en los Andes centrales son de tamaño pequeño a mediano, y habitantes de bofedales, riachuelos, ríos o sus orillas, que drenan finalmente hacia los principales sistemas hidrológicos (Fig. 1). En la cuenca del Titicaca se encuentra una especie grande con adaptaciones exclusivamente acuáticas en lagos y lagunas, Telmatobius culeus (Garman) (Vellard 1951, 1952; Hutchinson et al. 1976).

Por otro lado, Batrachophrynus está limitado a los Andes de Perú central (Fig. 1) con sólo dos especies: Batrachophrynus macrostomus Peters, la gran rana del lago Junín y de otras lagunas cercanas, y Batrachophrynus brachydactylus Peters de menor tamaño y habitante de ríachuelos que drenan al sistema hidrológico de los ríos Mantaro y Perené (Vellard 1952). La restringida distribución de Batrachophrynus y su similaridad morfológica y ecológica con Telmatobius, llevo a reconsiderar la validez del género Batrachophrynus y la discusión sobre sus relaciones de parentesco ha sido compleja (para más detalles ver Aguilar y Pacheco 2005, Córdova y Descailleaux 2005, Sinsch et al. 2005, Aguilar 2006). Ultimamente, análisis filogenéticos llevados a cabo con diferentes sistemas de caracteres han pro-

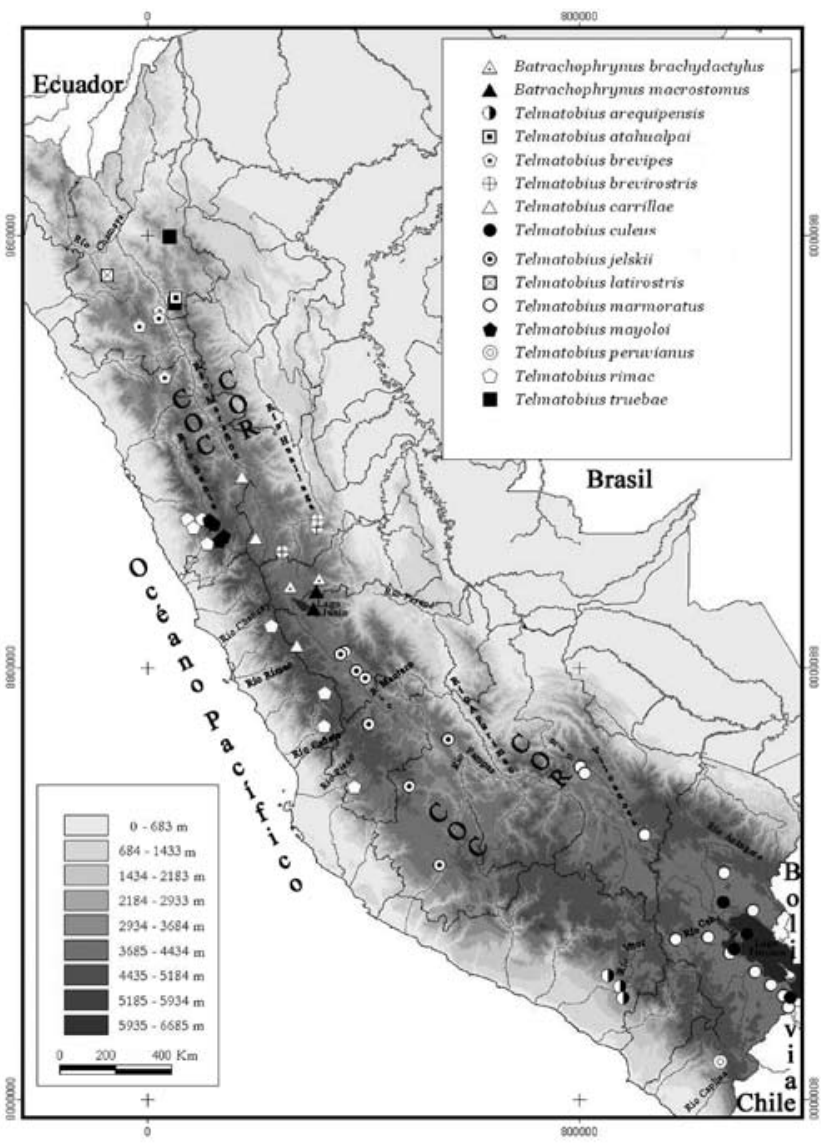

Figura 1. Mapa del Perú con las localidades de las especies de Batrachophrynus y Telmatobius utilizadas en este estudio. COC= Cordillera Occidental; COR= Cordillera Oriental. 
puesto relaciones estrechas entre Telmatobius y Batrachophrynus (Aguilar y Pacheco 2005, Córdova y Descailleaux 2005, Sinsch et al. 2005). Aguilar y Pacheco (2005) como Córdova y Descailleaux (2005) indican que Batrachophrynus está estrechamente relacionado a Telmatobius y ubican a $B$. macrostomus basal con respecto a Telmatobius, y a $B$. brachydactylus más relacionado con las especies de Telmatobius que con B. macrostomus. Sinsch et al. (2005) también apoyan una estrecha relación entre $B a$ trachophrynus y Telmatobius, pero Batrachophrynus junto con Telmatobius carrillae Morales forman un clado separado de las restantes especies de Telmatobius. Frost et al. (2006) no apoya una relación estrecha entre Telmatobius y Batrachophrynus, y los clasifica en dos familias con relaciones muy lejanas entre sí, Ceratophryidae y Batrachophrynidae. Posteriormente Frost (2009) incorpora a Batrachophrynus dentro de Telmatobiinae (Ceratophryidae) junto con Telmatobius. Aguilar (2006) sinonimiza Batrachophrynus y Telmatobius, pero no utiliza a ningún Ceratophryidae como grupos externos.

Los objetivos de este estudio son reevaluar las relaciones entre Batrachophrynus y Telmatobius, sugerir posibles sinapomorfías para Telmatobiinae, y discutir las implicancias del análisis filogenético.

\section{Material y métodos}

Se utilizaron 234 especímenes adultos y juveniles, y 173 larvas que pertenecen al Departamento de Herpetología del Museo de Historia Natural de la Universidad Nacional Mayor de San Marcos (MUSM), Lima, Perú, excepto los condrocráneos de $B$. brachydactylus que pertenecen al Museo de Historia Natural de la Universidad Ricardo Palma (MHNURP), Lima, Perú (Apéndice 1). Los estadios larvales se determinaron siguiendo a Gosner (1960) y se muestran en la Tabla 1. Las larvas fueron disectadas para examinar las cavidades bucofaríngeas y éstas se tiñieron con una solución de azul de metileno siguiendo a Wassersug (1976). Los esqueletos larvales y los cráneos de adultos fueron teñidos siguiendo la técnica de Dingerkus y Uhler (1977). Se contaron las espículas que estuvieran en un cuadrante de $1 \mathrm{~mm}^{2}$ y a una magnificación de 50x siguiendo a Sinsch et al. (2005). La terminología de los músculos de la mano sigue a Burton (1998). Todas las disecciones, observaciones y mediciones se hicieron con asistencia de un microscopio estereoscópico con cámara lúcida.

Tabla 1. Estadios de Gosner (1960) de las larvas examinadas.

\begin{tabular}{ll}
\multicolumn{1}{c}{ Especie } & \multicolumn{1}{c}{ Estadios de Gosner } \\
\hline Alsodes gargola & $36,38,40$ \\
Batrachophrynus brachydactylus & $34-37,39,40$ \\
Batrachophrynus macrostomus & $32,34-39$ \\
Telmatobius arequipensis & $35,36,38-42$ \\
Telmatobius atahualpai & $35,36,38$ \\
Telmatobius brevipes & $36-41$ \\
Telmatobius brevirostris & $31-33,35,38,40$ \\
Telmatobius carrillae & $34-41$ \\
Telmatobius culeus & $32-41$ \\
Telmatobius jelskii & $30-35,38,40$ \\
Telmatobius latirostris & 27,30 \\
Telmatobius marmoratus & $34-38,40,41$ \\
Telmatobius mayoloi & $30-32,35,37,39$ \\
Telmatobius peruvianus & $32,36,40$ \\
Telmatobius rimac & $34-36,38,39$ \\
Telmatobius truebae & $27,30-34,36,37,38,39$ \\
\hline
\end{tabular}

La polaridad de los estados de carácter se determinó por el método del grupo externo. Se utilizaron para este propósito a Gastrotheca peruana (Boulenger, 1900), Alsodes gargola Gallardo, 1970, Atelognathus patagonicus (Gallardo 1962) y Chacophrys pierotti (Vellard 1948). De los grupos externos mencionados, Gastrotheca peruana (Hemiphractidae) es el más distante y por lo tanto fue utilizado para polarizar los caracteres y enraizar los cladogramas resultantes. El siguiente grupo externo menos distante es Alsodes gargola (Cicloramphidae) que forma parte de Hesticobatrachia, el grupo hermano de Ceratophryidae (Frost et. al. 2006, Grant et al. 2006). Se escoge a Atelognathus patagonicus porque forma parte de Batrachylinae (Ceratophryidae) el grupo hermano de Ceratophryinae y Telmatobiinae (Grant et al. 2006). Se selecciona también a Chacophrys pierotti porque es parte de Ceratophryinae (Ceratophryidae), el grupo hermano de Telmatobiinae formado a su vez por Batrachophrynus y Telmatobius (Frost et al. 2006; Grant et al. 2006).

El grupo interno lo forman 15 especies de Telmatobiinae distribuidas en los Andes centrales. Los Andes centrales, siguiendo la división de los Andes usada por Torres-Carvajal (2007), es aquella ubicada entre la depresión de Huancabamba al norte de Perú y el cinturón de Arica al norte de Chile. Se incluyen en el grupo interno las dos especies de Batrachophrynus (B. brachydactylus y B. macrostomus) y 13 especies de Telmatobius (Telmatobius arequipensis Vellard, Telmatobius atahualpai Wiens, Telmatobius brevipes Vellard, Telmatobius brevirostris Vellard, Telmatobius carrillae Morales T. culeus, Telmatobius jelskii (Peters), Telmatobius latirostris Vellard, Telmatobius marmoratus (Dumeril y Bribon), Telmatobius mayoloi Salas y Sinsch, Telmatobius peruvianus Wiegmann, Telmatobius rimac Schmidt, y Telmatobius truebae Wiens). Las localidades de las especies examinadas se muestran en la figura 1.

La descripción de los caracteres y sus estados se muestra en la Tabla 2. Los estados de caracter están no ordenados (excepto los caracteres 0 y 15) y todos los caracteres tienen el mismo peso. Los estados de carácter que no se pudieron determinar se codificaron con "?". Los caracteres polimórficos se codificaron con los estados correspondientes al taxón respectivo y encerrado entre corchetes. Los estados del carácter 22 (frontoparietales) se tomaron principalmente de la literatura (Lynch 1978, Wiens 1993, Sinsch et al. 2005). Los estados de carácter para Atelognathus patagonicus y Chacophrys pierotti se obtuvieron de la literatura (Lynch 1978, Cei 1980, Lavilla 1988, Wassersug y Heyer 1988, Faivovich y Carrizo 1992, Burton 1998, Echevarría et al. 2006, Fabrezi 2006, Quinzio et al. 2006). En la Tabla 3 se presenta la matriz de caracteres y taxones.

El análisis filogenético, optimización de los estados de carácter, cladogramas y valores de soporte de los clados se obtuvieron con el programa TNT versión 1.1 (Goloboff et al. 2003b). Los métodos de enumeración implícita y búsqueda tradicional en TNT fueron usados para identificar todos los posibles árboles parsimoniosos con sus longitudes. En el método de búsqueda tradicional, los árboles de menor longitud fueron encontrados por el algoritmo de Wagner con 10 replicaciones (10 secuencias de adición al azar), sometidos luego al algoritmo de permutación de ramas (branch swapping) TBR (tree bisection reconnection) y guardando como máximo 10 árboles por replicación. La búsqueda de los árboles se hizo colapsando todos los nodos cuya longitud mínima es cero y sólo se consideraron las sinapomorfías compartidas por todos los árboles. 
Tabla 2. Lista de 24 carácteres y estados de carácter usados en este estudio

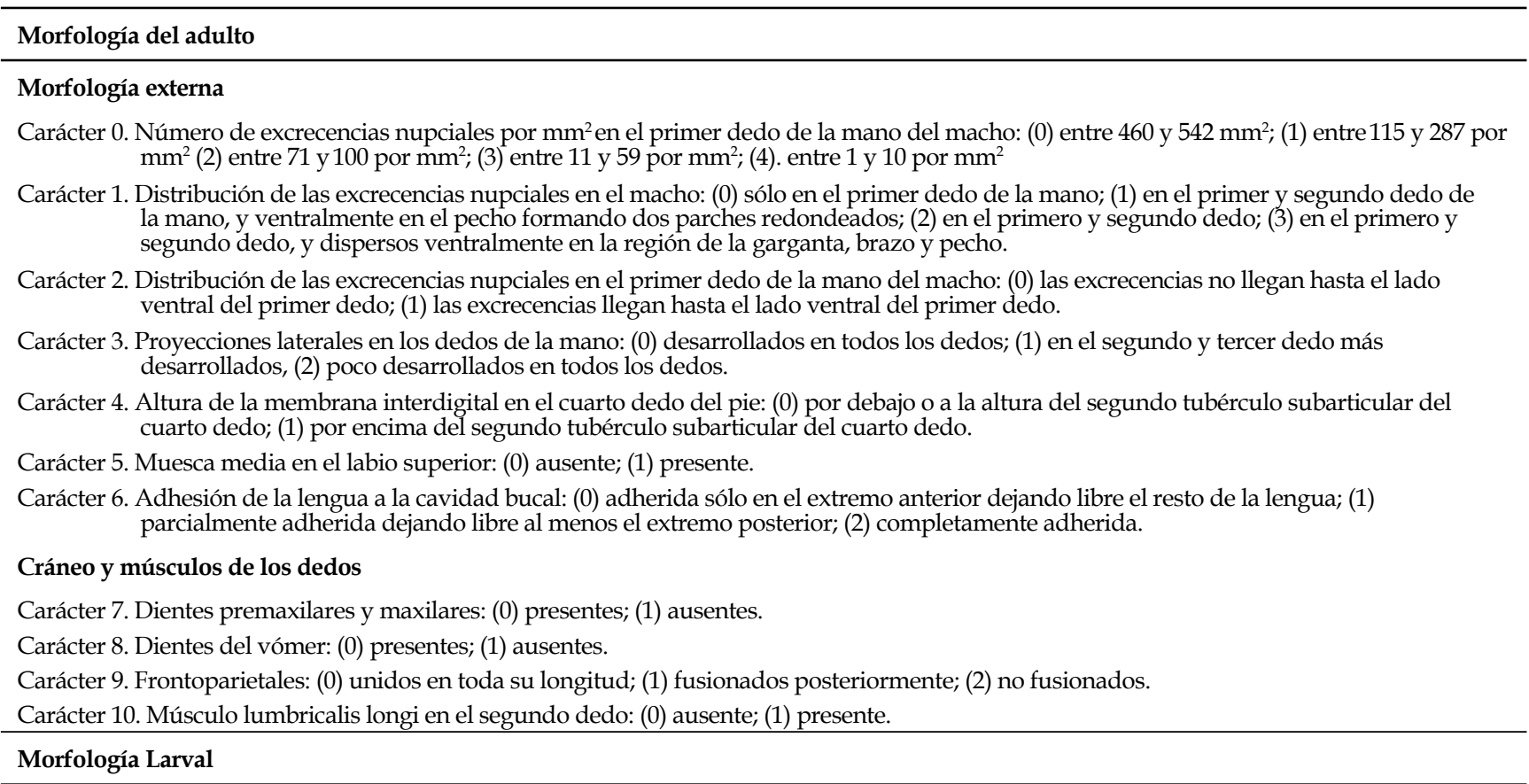

\section{Disco oral}

Carácter 11. Márgenes laterales del disco oral: (0) emarginado; (1) no emarginado.

Carácter 12. Distribución de las papilas marginales del disco oral: (0) ausentes anteriormente y presentes posteriormente; (1) presentes en todo el borde del disco oral

Carácter 13. Distribución de las papilas submarginales laterales del disco oral: (0) papilas presentes en el labio posterior; (1) papilas presentes en el límite de los labios anterior y posterior; (2) papilas presentes en ambos labios pero no forman una hilera continua; (3) papilas forman una hilera continua en ambos labios

Carácter 14. Papilas submarginales posteriores: (0) ausente; (1) presente.

Carácter 15. Número de filas de dentículos labiales: (0) dos anteriores y tres posteriores; (1) dos anteriores y cuatro posteriores; (2) tres anteriores y seis posteriores

\section{Cavidad bucofaríngea}

Carácter 16. Papilas infralabiales laterales del piso de la cavidad bucal: (0) bifurcados desde su base y cada bifurcación en forma de cono simple: (1) en forma de cono simple; (2) en forma de palpo aplanado con proyecciones en su borde libre.

Carácter 17. Número de papilas linguales: (0) Cuatro o más papilas; (1) una a tres papilas.

Carácter 18. Proyecciones en la arena prenarial: (0) En forma de una cresta y con proyecciones que salen de la cresta; (1) en forma de una papila; (2) ausentes; (3) en forma de pústulas pequeñas y no fusionadas.

Carácter 19. Proyecciones del velum dorsal: (0) Ausentes; (1) proyecciones poco desarrolladas; (2) proyecciones desarrolladas.

\section{Condrocráneo}

Carácter 20. Plano formado por el extremo anterior de los cuernos trabeculares: (0) no es perpendicular al eje axial; (1) Perpendicular al eje axial. Carácter 21. Cuerpos del suprarrostral: (0) fusionado; (1) no fusionado.

Carácter 22. Comisura cuadrado-orbital: (0) Presente; (1) ausente.

Carácter 23. Relación entre el margen anterior de la cápsula ótica y el proceso ascendente del palatocuadrado: (0) cercana; (1) separada.

Carácter 24. Dirección del proceso ascendente del palatocuadrado al eje axial del condrocráneo: (0) no perpendicular; (1) perpendicular.

Para hallar los valores de soporte de los clados resultantes, la matriz fue analizada con el método de Bootstrap (Felseinstein, 1985), pero tomando en cuenta la influencia de los caracteres irrelevantes y haciendo independiente la probabilidad del pesado de cada carácter (Harshman, 1994; Goloboff et al., 2003a). El remuestreo incluyó 1000 réplicas y la matriz fue analizada mediante búsqueda tradicional. Para evitar los problemas asociados al uso de frecuencias absolutas como medida de soporte, los valores de Bootstrap que se muestran son la diferencia entre la frecuencia de un grupo presente en el cladograma y la frecuencia del grupo que más lo contradice (valores GC) (Goloboff et al. 2003a). Los valores GC varían entre -1 y 1 indicando, respectivamente, máxima contradicción y máximo soporte. Los valores GC del Bootstrap se muestran en porcentajes. Debido a que resultó más de un árbol en el análisis, se construyó un árbol de consenso estricto.

\section{Resultados}

Los métodos de enumeración implícita y búsqueda tradicional usando 25 caracteres de la morfología de la larva y adulto resultaron en 20 árboles igualmente parsimoniosos de 56 pasos. El consenso estricto de estos árboles con los valores de soporte GC de Bootstrap se muestra en la figura 2.

Las dos especies de Batrachophrynus están estrechamente relacionadas, pero forman parte de un clado constituido también por T. carrillae y T. mayoloi. Las sinapomorfías que apoyan la unión de Batrachophrynus, T. carrillae y T. mayoloi son la presencia de entre 115 y 287 espículas nupciales por milimetro cuadrado en el primer dedo de la mano, excrecencias nupciales llegan hasta el lado ventral del primer dedo, lengua completamente adherida a la cavidad bucal, y frontoparietales no fusionados. Este grupo monofilético tiene un valor de Bootstrap de 48. 
Tabla 3. Matriz de caracteres y taxones para Gastrotheca peruana, Alsodes gargola, Atelognathus patagonicus, Chacophrys pierotti, 2 especies de Batrachophrynus y 13 especies de Telmatobius.

\begin{tabular}{|c|c|c|c|c|c|c|c|c|c|c|c|c|c|c|c|c|c|c|c|}
\hline \multirow[b]{2}{*}{ Uี } & \multicolumn{19}{|c|}{ Taxon } \\
\hline & 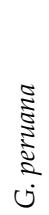 & $\begin{array}{l}\frac{5}{0} \\
0 \\
\vdots \\
0 \\
\dot{\leftarrow}\end{array}$ & 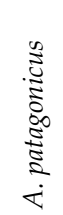 & 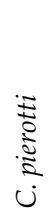 & 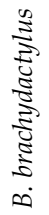 & 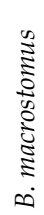 & 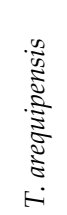 & 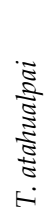 & 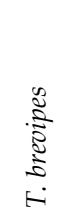 & 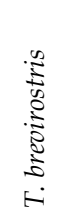 & 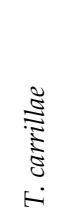 & $\begin{array}{c}\frac{0}{3} \\
\frac{\Xi}{J} \\
\text { H }\end{array}$ & $\frac{: \tilde{J}}{\frac{\tilde{D}}{2}}$ & 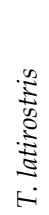 & 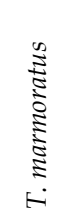 & 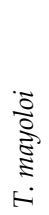 & 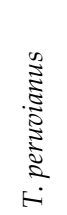 & 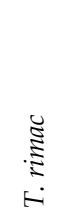 & $\begin{array}{l}\frac{\Xi}{\Xi} \\
\stackrel{\Xi}{\Xi} \\
\stackrel{ \pm}{ \pm} \\
-\end{array}$ \\
\hline 0 & 0 & 4 & $?$ & $?$ & 1 & 1 & 4 & 4 & 2 & 4 & 1 & 3 & 3 & 4 & 3 & 1 & 3 & [12] & 4 \\
\hline 1 & 0 & 1 & 2 & $?$ & 0 & 0 & 3 & 0 & 0 & [03] & 0 & 0 & 3 & 0 & [03] & 0 & [03] & 0 & 0 \\
\hline 2 & 0 & 0 & 0 & $?$ & 1 & 1 & 1 & 0 & 1 & 0 & 1 & 1 & 1 & 0 & 1 & 1 & 1 & 1 & 0 \\
\hline 3 & 0 & 0 & 2 & 2 & 2 & 2 & 1 & 2 & 2 & 2 & 2 & 1 & [12] & 2 & 1 & 2 & 1 & 2 & 2 \\
\hline 4 & 0 & 0 & 1 & 0 & 0 & 0 & 0 & 0 & 0 & 1 & 0 & 0 & 0 & 0 & 0 & 0 & 0 & [01] & 0 \\
\hline 5 & 0 & 0 & 0 & 0 & 0 & 0 & [01] & 0 & 0 & 0 & 0 & 1 & [01] & 0 & 1 & 0 & [01] & 0 & 0 \\
\hline 6 & 0 & 0 & 1 & 0 & 2 & 2 & 1 & 1 & 1 & 1 & 2 & 1 & 1 & 1 & [12] & 2 & 1 & 1 & 1 \\
\hline 7 & 0 & 0 & [01] & 0 & 1 & 1 & 0 & 0 & 0 & 0 & 1 & 0 & 0 & 0 & 0 & 0 & 0 & 0 & 0 \\
\hline 8 & 0 & 0 & [01] & 0 & 1 & 1 & [01] & 0 & 0 & 0 & 1 & [01] & [01] & 0 & [01] & 0 & [01] & 0 & 0 \\
\hline 9 & 0 & 2 & 2 & 0 & 2 & 2 & 1 & 1 & 1 & ? & [12] & 1 & 1 & 1 & 1 & 2 & 1 & 1 & [12] \\
\hline 10 & 0 & 0 & 0 & 0 & 1 & 1 & [01] & 0 & 0 & [01] & [01] & 0 & 0 & 0 & [01] & 0 & 0 & [01] & [01] \\
\hline 11 & 0 & 0 & 0 & 0 & 1 & 1 & 1 & 1 & 1 & 1 & 1 & 1 & 1 & 1 & 1 & 1 & 1 & 1 & 1 \\
\hline 12 & 0 & 0 & 0 & 1 & 0 & 0 & 0 & 1 & 0 & [01] & 0 & 0 & 0 & 0 & 0 & 0 & 0 & 0 & 0 \\
\hline 13 & 0 & 2 & 2 & 1 & 3 & 3 & 3 & 0 & 3 & [03] & 3 & 3 & 3 & 3 & 3 & 3 & 3 & 3 & 3 \\
\hline 14 & 0 & 1 & 0 & 0 & 0 & 0 & 0 & 1 & 0 & [01] & 0 & 0 & 0 & 0 & 0 & 0 & 0 & [01] & 0 \\
\hline 15 & 0 & 0 & 0 & 0 & 0 & 0 & 0 & 2 & 0 & [01] & 0 & 0 & 0 & 0 & 0 & 0 & 0 & 0 & 0 \\
\hline 16 & 0 & 1 & 1 & $?$ & 2 & 2 & 2 & 2 & 2 & 2 & 2 & 2 & 2 & 2 & 2 & 2 & 2 & 2 & 2 \\
\hline 17 & 0 & 0 & 0 & $?$ & 1 & 1 & 1 & 1 & 1 & 1 & 1 & 1 & 1 & 1 & 1 & 1 & 1 & 1 & 1 \\
\hline 18 & 0 & 1 & 2 & $?$ & 2 & 3 & 2 & 2 & 2 & 2 & 2 & 2 & 2 & 2 & 2 & 2 & 2 & 2 & 2 \\
\hline 19 & 0 & 1 & 1 & $?$ & 1 & 1 & [12] & 1 & [12] & 1 & 1 & 1 & 1 & 1 & 1 & 1 & 2 & 2 & 1 \\
\hline 20 & 0 & 1 & $?$ & 0 & 0 & 0 & 0 & 0 & 0 & 0 & 0 & 0 & 0 & 0 & 0 & 0 & 0 & 0 & 0 \\
\hline 21 & 0 & 1 & $?$ & 0 & 1 & 1 & 1 & 0 & 1 & 1 & 1 & 1 & 1 & 1 & 1 & 1 & 1 & 1 & 1 \\
\hline 22 & 0 & 0 & ? & 0 & 1 & 1 & 1 & 1 & 1 & 1 & 1 & 1 & 1 & 1 & 1 & 1 & 1 & 1 & 1 \\
\hline 23 & 0 & 0 & $?$ & 1 & 0 & 1 & 0 & 0 & 0 & 0 & 0 & 0 & 0 & 0 & 0 & 0 & 0 & 0 & 0 \\
\hline 24 & 0 & 0 & ? & 1 & 1 & 1 & 1 & 0 & 1 & 1 & 0 & 1 & 1 & 0 & 1 & 0 & 1 & 0 & 0 \\
\hline
\end{tabular}

El grupo formado por Batrachophrynus y T. carrillae está sustentado por dos sinapomorfías, la ausencia de dientes premaxilares y maxilares, y ausencia de dientes vomerianos. Este clado tiene un valor de Bootstrap de 64 .

La monofilia de Batrachophrynus está apoyada por una sinapomorfía, la dirección del proceso ascendente del palatocuadrado al eje axial del condrocráneo es perpendicular. Este clado tiene un valor de Bootstrap de 43.

Otro grupo monofilético dentro del clado de Telmatobius y con relaciones no resueltas es el formado por T. arequipensis, $T$. culeus, T. jelkii, T. marmoratus y T. peruvianus. La sinapomorfía que los apoya es la presencia de excrecencias nupciales que llegan hasta el lado ventral del primer dedo, proyecciones laterales en el segundo y tercer dedo más desarrollados, muesca media en el labio superior, y dirección perpendicular del proceso ascendente del palatocuadrado al eje axial del condrocráneo. Este clado tiene un valor de Bootstrap de 21.

En el consenso estricto, las restantes especies de Telmatobius (T. atahualpai, T. brevipes, T. brevirostris, T. latirostris, T. rimac y $T$. truebae) tienen relaciones no resueltas.

El clado formado por todas las especies de Telmatobius (incluyendo a Batrachophrynus) está sustentado por presentar una lengua parcialmente adherida a la cavidad bucal dejando libre al menos el extremo posterior, frontoparietales fusionados posteriormente, márgenes laterales del disco oral no emarginados, papilas submarginales laterales forman una hilera continúa en los dos labios, papilas infralabiales laterales en forma de palpos aplanados con proyecciones en su borde libre, una a tres papilas linguales, y comisura cuadrado-orbital ausente. Este clado tiene un valor de Bootstrap de 36.

Las relaciones entre los grupos externos (Alsodes gargola, Atelognathus patagonicus y Chacophrys pierotti) no están resueltas.

\section{Discusión}

Este análisis filogenético confirma la hipótesis de que Telmatobius es parafilético en relación con Batrachophrynus, y que las especies de Batrachophrynus están más cercanamente relacionadas una con otra dentro de un clado formado por $T$. carrillae y T. mayoloi (Aguilar, 2006). Por lo tanto, se sinonimiza Batrachophrynus y Telmatobius. Resultados similares han sido obtenidos por un análisis filogenético de 30 especies de Telmatobius incluyendo a Batrachophrynus (Barrionuevo, en preparación). En este estudio, Batrachophrynus también se encuentra formando relaciones estrechas con T. carrillae y T. mayoloi, y este grupo monofilético formado por los 4 taxones es uno de los más derivados dentro de Telmatobius. La diferencia estriba en que $B$. macrostomus está más relacionado con $T$. carrillae que con $B$. brachydactylus (Barrionuevo, en preparación).

Los resultados de nuestro estudio y el de Barrionuevo sugieren que los caracteres que habían sido usados para distinguir a Batrachophrynus de Telmatobius (la ausencia de dientes premaxi- 
Figura 2. Consenso estricto de 2 árboles de 52 pasos. Los números arriba de las ramas son los caracteres y entre paréntesis los estados respectivos que apoyan los clados. Los números debajo de las ramas son los valores de Bootstrap.

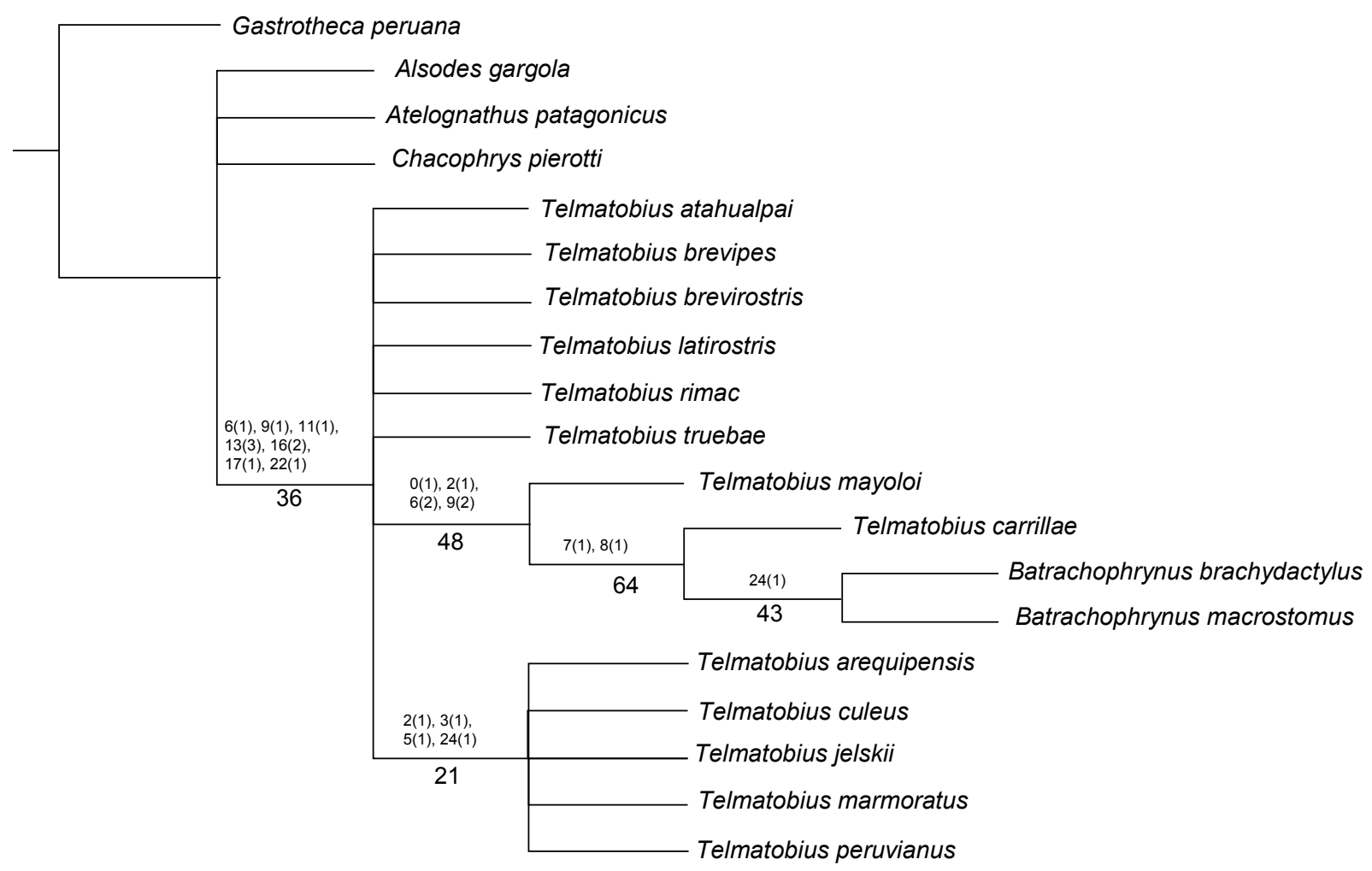

lares, maxilares y vomerianos, lengua completamente adherida y excrecencias nupciales diminutas) se puede interpretar más bien como reducciones que han ocurrido dentro de la historia evolutiva de Telmatobius (Sinsch et al. 2005).

Nuestro estudio también sugiere algunas sinapomorfías para Telmatobiinae. Por ejemplo, el disco oral no emarginado está presentes en todas las larvas conocidas de Telmatobius incluyendo aquellas que son reofilas como T. atahualpai, T. espadai y en algunos individuos de T. brevirostris (Diaz y Valencia 1985, Lavilla 1988, Lavilla y De la Riva 1993, Formas et al. 1999, De la Riva y Harvey 2003, Formas et al. 2003, De la Riva 2005, Formas et al. 2005, Lavilla y Barrionuevo 2005, Aguilar 2006, Aguilar et al. 2007a, 2007b, Vera Candioti 2008, Aguilar y Lehr 2009). Del mismo modo, las papilas infralabiales laterales en forma de palpos aplanados con proyecciones en su borde libre, una a tres papilas linguales, y comisura cuadrado-orbital ausente son estados de carácter presentes no sólo en las especies de los Andes centrales, sino también en algunos Telmatobius de los Andes del sur (Fabrezi y Lavilla 1993, Lavilla y De la Riva 1993, Aguilar y Pacheco 2005, Aguilar 2006, Aguilar et al. 2007b, Vera Candioti 2008).

\section{Agradecimientos}

El primer autor agradece de manera especial a Alejandro Aguilar. Jesús Córdova brindó espacio y acceso a los especimenes. A Mercedes Gonzales y Victor Morales del MHNURP por el préstamo de especimenes. A Victor Morales y Edgar Lehr por la revisión de una primera versión del manuscrito y por sus importantes contribuciones al mismo. A Karen Siu Ting por su coloboración en la elaboración del mapa. Paúl Velazco proporcionó valiosa literatura. Boris Blotto, Mikael Lundberg, Elías Ponce, Juana Suárez y Pablo Venegas apoyaron en la obtención de especimenes.

\section{Literatura Citada}

Aguilar C. \& V. Pacheco. 2005. Contribución de la morfología bucofaríngea larval a la filogenia de Batrachophrynus y Telmatobius. In: Lavilla, E. O., I. De la Riva, E. Font y J. Lluch (eds.). Estudios sobre las ranas andinas de los géneros Telmatobius y Batrachophrynus (Anura: Leptodactylidae). Monografías de Herpetología Vol. 7, Asociación Herpetológica Española, Valencia. p. 219-238.

Aguilar C. 2006. Relaciones filogenéticas entre algunos Telmatobinidos (Anura, Leptodactylidae, Telmatobiinae) de Perú basado en la morfología de los estados larval y adulto. Tesis, Magíster en Zoología, mención Sistemática y Evolución. Facultad de Ciencias Biológicas Universidad Nacional Mayor de San Marcos. http://www.cybertesis. edu.pe/sisbib/2006/aguilar_pc/html/index-frames.html. Acceso 19/02/2009

Aguilar C., K. Siu Ting \& P. Venegas. 2007a. The rheophilous tadpole of Telmatobius atahualpai (Anura: Ceratophrydae). South American Journal of Herpetology. 2(3): 165-174.

Aguilar C., M. Lundberg, K. Siu Ting \& M. Jiménez. 2007b. Nuevos registros para la la herpetofauna del departamento de Lima, descripción del renacuajo de Telmatobius rimac Schmidt, 1954 (Anura: Ceratophrydae) y una clave para los anfibios. Revista Peruana de Biología 14(2): 209-216.

Aguilar C. \& E. Lehr. 2009. The tadpole of Telmatobius mayoloi. Journal of Herpetology 43: 160-165.

Barrionuevo J. S \& D. Baldo. 2009. A new species of Telmatobius (Anura, Ceratophryidae) from Northern Jujuy Province, Argentina. Zootaxa 2030: 1-20.

Barrionuevo J. S. En Preparación. Análisis filogenético de las especies del grupo meridional del género Telmatobius (Anura: Leptodactylidae). Tesis Doctoral. Universidad Nacional de Tucumán, Argentina.

Burton T. C. 1998. Pointing the way: The distribution and evolution of some characteres of the finger muscles of frogs. American Museum Novitates 3229: 1-13. 
Cei J. M. 1980. Amphibians of Argentina. Monitore Zoologico. Nouva Serie. Monographia, 2:1-609.

Cei J. M. 1986. Speciation and adaptative radiation in Andean Telmatobius frogs. En: F. Vuilleumier and M. Monasterio (eds.). High altitude tropical biogeography, New York, Oxford Univ. Press. p. 374-386.

Córdova J. H. \& J. Descailleaux. 2005. El análisis cladístico preliminar de los cariotipos de cinco especies de Telmatobius y dos de Batrachophrynus no apoya su separación genérica. In: Lavilla, E. O., I. De la Riva, E. Font y J. Lluch (eds.). Estudios sobre las ranas andinas de los géneros Telmatobius y Batrachophrynus (Anura: Leptodactylidae). Monografías de Herpetología Vol. 7, Asociación Herpetológica Española, Valencia. p. 187-218.

Czopeck J. 1983. Distribution of capillaries in the repiratory surfaces in two species of Batrachophrynus (Amphibia, Anura, Leptodactylidae). Zoologica Poloniae 30:211-224.

De la Riva I. \& M.B Harvey. 2003. A new species of Telmatobius from Bolivia and a redescription of T. simonsi Parker, 1940 (Amphibia: Anura: Leptodactylidae). Herpetologica 59: 127-142.

De la Riva I. 2005. Sinopsis of Bolivian Telmatobius. In: Lavilla, E. O., I. De la Riva, E. Font y J. Lluch (eds.). Estudios sobre las ranas andinas de los géneros Telmatobius y Batrachophrynus (Anura: Leptodactylidae). Monografías de Herpetología Vol. 7, Asociación Herpetológica Española, Valencia. p. 65-101.

De Macedo H. 1950. Anotaciones para el conocimiento zoológico del género Batrachophrynus (Amphibia: Salientia). Tesis para optar el grado de Bachiller. Universidad Nacional Mayor de San Marcos, Lima. 27 p.

De Macedo H. 1976. Aspectos ecomorfológicos de los aparatos respiratorios y circulatorios en anfibios y andinos. Tesis para optar el grado de Doctor en Ciencias. Universidad Nacional Mayor de San Marcos, Lima. 51 p.

Diaz N. F. \& J. Valencia. 1985. Larval morphology and phenetic relationships of the Chilean Alsodes, Telmatobius, Caudiverbera and Insuetophrynus (Anura: Leptodactylidae). Copeia 1: 175-181.

Dingerkus G. \& D. Uhler. 1977. Enzyme clearing of alcian blue stained whole small vertebrates for demonstration of cartilage. Stain Technology 52: 229-232.

Echevarría D., C. A. Ubeda \& N. G. Basso. 2006. Scanning electron microscopy of the oral apparatus and buccopharyngeal cavity of Atelognathus salai larvae (Anura, Neobatrachia). Phyllomedusa 5: 77-81.

Fabrezi M. \& E. Lavilla. 1993. Anatomía del condrocráneo en larvas de tres especies de Telmatobius del grupo meridional (Anura: Leptodactylidae). Physis 48: 39-46.

Fabrezi M. 2006. Morphological evolution of Ceratophryinae (Anura, Neobatrachia). Journal of zoology 44: 153-166.

Faivovich J. \& G. R. Carrizo. 1992. Descripción de la larva de Chacophrys pierottii (Vellard, 1948) (Leptodactylidae, Ceratophryinae). Alytes, 10:81-89

Felseinstein J. 1985. Confidence limits on phylogenies: an approach using bootstrap 39: 783-791.

Formas R., I. Northland, J. Capetillo, J. J. Nuñez, C. Cuevas y L. M. Brieva. 1999. Telmatobius dankoi, una nueva especie de rana acuática del norte de Chile (Leptodactylidae). Revista Chilena de Historia Natural 72: 427-445.

Formas R., E. Benavides \& C. Cuevas. 2003. A new species of Telmatobius (Anura: Leptodactylidae) from río Vilama, northern Chile, and the redescription of T. halli Noble. Herpetologica 59: 253-270.

Formas R., A. Veloso \& J. C. Ortiz. 2005. Sinopsis de los Telmatobius de Chile. In: Lavilla, E. O., I. De la Riva, E. Font y J. Lluch (eds.). Estudios sobre las ranas andinas de los géneros
Telmatobius y Batrachophrynus (Anura: Leptodactylidae). Monografías de Herpetología Vol. 7, Asociación Herpetológica Española, Valencia. p. 103-114.

Frost D.R., T. Grant, J. Faivovich, R.H. Bain, A. Haas, et al. 2006. The amphibian tree of life. Bulletin American Museum of Natural History $N^{\circ} 297$. p. 1-370.

Frost D.R. 2009. Amphibian Species of the World: an Online Reference. Version 5.3 (12 February, 2009). Electronic Database accessible at http://research.amnh.org/herpetology/amphibia/American Museum of Natural History, New York, USA. Acceso 11/08/2009.

Goloboff P., J. S. Farris, M. Källersjö, B. Oxelman, M. Ramirez y C. A. Szumick. 2003a. Improvements to resampling measures of group support. Cladistics 19: 324-332.

Goloboff P., J. S. Farris \& K. Nixon. 2003b. T.N.T. Tree analysis using new technology. Version 1.0 (Beta 1, Oct 03).

Gosner K. L. 1960. A simplified table for staging anuran embryos and larvae with notes on identification. Herpetologica 16:183-190.

Grant T. D. Frost, J. P. Caldwell, R. Gagliardo, C. F. B. Haddad, et al. 2006. Phylogenetics systematics of Dart-Poison Frogs and their relatives (Amphibia: Athesphatanura: Dendrobatidae). Bulletin of the American Museum of Natural History 299: $262 \mathrm{pp}$.

Harshman J. 1994. The effect of irrelevant characters on bootstrap values. Systematic Biology 43: 419-424.

Hutchison V. H., H. B. Haines \& G. Engbretson. 1976. Aquatic life at high altitude. Respiratory adaptations in the lake Titicaca frog, Telmatobius culeus.

Lavilla E. O. 1988. Lower Telmatobiinae (Anura: Leptodactylidae): Generic diagnosis based on larval characters. Occasional Papers Museum Natural History University Kansas 124: 1-19.

Lavilla E. O. \& I. De la Riva. 1993. La larva de Telmatobius bolivianus (Anura. Leptodactylidae). Alytes 11: 37-46.

Lavilla E. O. \& J. S. Barrionuevo. 2005. El género Telmatobius en la República Argentina: una síntesis. Font y J. Lluch (eds.). Estudios sobre las ranas andinas de los géneros Telmatobius y Batrachophrynus (Anura: Leptodactylidae). Monografías de Herpetología Vol. 7, Asociación Herpetológica Española, Valencia. p. 115-165.

Lynch J. D. 1978. A re-assesment of the telmatobiine leptodactylid frogs of Patagonia. Occasional Papers Museum Natural History University of Kansas 72:1-57.

Quinzio S. I., M. Fabrezi, \& J. Faivovich. 2006. Redescription of the tadpole of Chacophrys pierottii (Vellard, 1948) (Anura:Ceratophryidae). South American Journal of Herpetology 1:202-209.

Seimon T., A. Seimon, P. Daszakz, S. Halloy, L. Schloegelz, et al. 2007. Upward Range Extension of Andean Anurans and Chytridiomycosis to Extreme Elevations in Response to Tropical Deglaciation. Global Change Biology 13: 288-299.

Sinsch U, Hein, K., \& B. Glump. 2005. Reassessment of central Peruvian Telmatobiinae (genera Batrachophrynus and Telmatobius): osteology, palmar morphology and skin histology. In: Lavilla, E. O., I. De la Riva, E. Font y J. Lluch (eds.). Estudios sobre las ranas andinas de los géneros Telmatobius y Batrachophrynus (Anura: Leptodactylidae). Monografías de Herpetología Vol. 7, Asociación Herpetológica Española, Valencia. p. 239-260.

Torres-Carvajal O. 2007. Phylogeny and biogeography of a large radiation of Andean lizards (Iguania, Stenocercus). Zoologica Scripta 36: 311-326

Trueb L. 1979. Leptodactylid frogs of the genus Telmatobius in Ecuador with the description of a new species. Copeia 4: 714-733. 
Vellard J. 1951. Estudios sobre batracios andinos. I. El grupo Telmatobius y formas afines. Memorias del Museo de Historia Natural "Javier Prado" 1: 1-89.

Vellard J. 1952. Adaptation des batraciens à la vie à grande hauteur dans les Andes. Bulletin de la Société Zoologique de France 77: 169-187.

Vera Candioti M. F. 2008. Larval Anatomy of Andean tadpoles of Telmatobius (Anura: Ceratophrydae) from Northwestern Argentina. Zootaxa 1938: 40-60.
Wassersug R. J. 1976. Oral morphology of anuran larvae: terminology and general description. Occasional Papers Museum Natural History University of Kansas 48:1-23.

Wassersug R. J. \& W. R. Heyer. 1988. A survey of internal oral features of leptodactyloid larvae (Amphibia: Anura). Smithsonian Contribution to Zoology 457:1-99.

Wiens J. J. 1993. Systematics of the leptodactylid frog genus Telmatobius in the Andes of northern Peru. Occasional Papers Museum Natural History University Kansas 162:1-76.

Apéndice 1. Especimenes examinados.

\begin{tabular}{|c|c|c|}
\hline Especie & Número MUSM & Localidad \\
\hline \multirow{3}{*}{ Alsodes gargola } & 19517 & $\begin{array}{l}\text { Pampa de Lonco Luan, Dto. Aluminé, } \\
\text { Neuquén, Argentina }\end{array}$ \\
\hline & 19518 & $\begin{array}{l}\text { Próx. Copahue, Dto. Ñorquín, Neuquén, } \\
\text { Argentina }\end{array}$ \\
\hline & 19519 (larvas), 19520 (larvas) & $\begin{array}{l}\text { Cerro Perito Moreno, Dto. Bariloche, Río } \\
\text { Negro, Argentina }\end{array}$ \\
\hline \multirow{5}{*}{ Batrachophrynus brachydactylus } & $\begin{array}{l}\text { 0088, 0098, 0104, 0112, 0449, 0452, 0470, 0489, 0492, 0493, } \\
0494,0497,0498,0488,0491,0490,0495,1334,7115,11060, \\
11032,11039\end{array}$ & Lago Junín, Dpto. de Junín, Perú \\
\hline & 10092 (larvas) & Capillacocha, Dpto. de Pasco, Perú \\
\hline & 11068 (larvas), 11075 (larvas), 10092 (larvas) & Ondores, Dpto. de Pasco, Perú \\
\hline & 10087 (larvas) & Riachuelo Añascancha, Dpto. de Pasco, Perú \\
\hline & MHNURP 023-A (larvas) & Río Huaylamayo, Dpto. de Pasco, Perú \\
\hline \multirow[t]{2}{*}{ Batrachophrynus macrostomus } & $\begin{array}{l}\text { 0001, 0009, 0016, 0039, 0044, 0049, 0061, 0118, 0250, 0253, } \\
0266,0292,0293,0294,0295,0296,0342 \text { (larvas), 0374, 0375, } \\
0376,0474,0477,16535 \text { (larvas), } 18535 \text { (larvas), } 18536\end{array}$ & Lago Junín, Dpto. de Junín, Perú \\
\hline & 1103 (larvas) & Laguna Capillacocha, Dpto. de Pasco, Perú \\
\hline \multirow{5}{*}{ Telmatobius arequipensis } & $3903,3904,3906,3907,3910,3911,3913,3914$ & $\begin{array}{l}\text { Riachuelo Characato, Dpto. de Arequipa, } \\
\text { Perú }\end{array}$ \\
\hline & 19228 (larvas) & Polobaya, Dpto. de Arequipa, Perú. \\
\hline & $3915,3916,3917,3918$ & Arequipa, Dpto. de Arequipa, Perú \\
\hline & $6774,6775,6776$ & Yura, Dpto. de Arequipa, Perú \\
\hline & $12577,12578,12579$ & Dpto. de Arequipa, Perú \\
\hline \multirow{3}{*}{ Telmatobius atahualpai } & $15976,15978,15979,15980,15984$ & Río Abiseo, Dpto. de San Martin, Perú \\
\hline & 19470, 19478, 19479, 19499, 22776 (larvas) & Leimebamba, Dpto. de Amazonas, Perú \\
\hline & 19602 & $\begin{array}{l}\text { Laguna Quintecocha, Dpto. de San Martín, } \\
\text { Perú }\end{array}$ \\
\hline \multirow{5}{*}{ Telmatobius brevipes } & $3740,3742,3743,3744,3749,6186$ & Huamachuco, Dpto. de La Libertad, Perú \\
\hline & 6301 (larvas) & Cajabamba, Dpto. de La Libertad, Perú. \\
\hline & 6280 (larvas) & Cajamarca, Dpto. de Cajamarca, Perú \\
\hline & 19315 (larvas) & Sanagoran, Dpto. de La Libertad, Perú \\
\hline & 19314 (larvas) & Río Negro, Dpto. de La Libetad, Perú \\
\hline \multirow{4}{*}{ Telmatobius brevirostris } & $7666,7667,7669$ & Caina, Ambo, Dpto. de Huánuco, Perú \\
\hline & 7676,7677 & Chasqui, Ambo, Dpto. de Huánuco, Perú \\
\hline & 20468,20469 & Tomayrica, Dpto. de Huánuco, Perú \\
\hline & 20464, 20466, 20547 (larvas) & Chaglla, Dpto. de Huánuco, Perú \\
\hline \multirow{4}{*}{ Telmatobius carrillae } & $1528,1544,1545,3932,3933,3934$ & Yuraccyacu, Dpto. de Ancash, Perú \\
\hline & 5622 (larvas), 5639 (larvas) & Chopitec, Dpto. de Ancash, Perú \\
\hline & 5662 (larvas) & Rumi Rajra, Dpto. de Ancash, Perú \\
\hline & $6661,6664,6667,6672,6680,6681,6682,6683,6684$ & Huikia, Dpto. de Ancash, Perú \\
\hline
\end{tabular}

Continúa... 
Apéndice 1....

\begin{tabular}{|c|c|c|}
\hline \multirow{8}{*}{ Telmatobius culeus } & $7767,7789,7816,7817,7820,7821,7822$ & Río Juliaca, Dpto. de Puno, Perú \\
\hline & 7818 (larvas), 7819 (larvas) & Lago Titicaca, Dpto. de Puno, Perú \\
\hline & 7769,7770 & Isla del Sol, Bolivia \\
\hline & $7786,7792,7806,7823,7824$ & Lagunillas, Dpto. de Puno, Perú \\
\hline & 7771,7772 & Río Coata, Juliaca, Dpto. de Puno, Perú \\
\hline & 7755 & Río Ilave, Chicuito, Dpto. de Puno, Perú \\
\hline & $12296,12297,12298,12299$ & Desaguadero, Dpto. de Puno, Perú \\
\hline & $12565,12566,12567$ & Saracocha, Dpto. de Arequipa, Perú \\
\hline \multirow{9}{*}{ Telmatobius jelskii } & $7639,7640,7641$ & Huancavelica, Dpto. de Huancavelica, Perú \\
\hline & 7112 (larvas) & Chancas, Dpto. de Junín, Perú \\
\hline & 18499 (larvas) & Cangallo, Dpto. de Ayacucho, Perú \\
\hline & 18497 (larvas) & Quebrada Cachi, Dpto. de Ayacucho, Perú \\
\hline & 18496 (larvas) & Rio Yucay, Dpto. de Ayacucho, Perú \\
\hline & $7646,7647,7648,7649,7650,7651$ & Tambo, La Mar, Dpto. de Ayacucho, Perú \\
\hline & $16862,16883,16865$ & Huancayo, Dpto. de Junín, Perú \\
\hline & $16851,16769,16773,16786$ & Jauja, Dpto. de Junín, Perú \\
\hline & 12907,12909 & Parinacochas, Dpto. de Ayacucho, Perú \\
\hline \multirow{3}{*}{ Telmatobius latirostris } & $3730,3731,3733,3734,3735,3736,3738,7866$ & Cutervo, Dpto. de Cajamarca, Perú \\
\hline & 17125 (larvas) & $\begin{array}{l}\text { San Andrés de Cutervo, Dpto. de } \\
\text { Cajamarca, Perú }\end{array}$ \\
\hline & 0960 & Chorro Blanco, Dpto. de Cajamarca, Perú \\
\hline \multirow{12}{*}{ Telmatobius marmoratus } & $7689,7690,7691,7692,7693$ & $\begin{array}{l}\text { Laguna Chincheros, Urubamba, Dpto. de } \\
\text { Cuzco, Perú }\end{array}$ \\
\hline & 17026 (larvas) & Puno, Dpto. de Puno, Perú \\
\hline & 19603 (larvas) & Platería, Dpto. de Puno, Perú \\
\hline & 19480 (larvas) & Pumachanca, Dpto. de Cuzco, Perú \\
\hline & $7758,7764,7765,12373$ & Bahía de Juli, Pomata, Dpto. de Puno, Perú \\
\hline & 12896 & Tinta, Dpto. de Cuzco, Perú \\
\hline & $12014,12015,12024,12267,12302$ & Huancurcuchu, Dpto. de Puno, Perú \\
\hline & $7687,7688,12323$ & Urubamba, Cuzco \\
\hline & $3920,3925,3927$ & Calacoto, la Paz, Bolivia \\
\hline & 12342,17005 (larvas) & Desaguadero, Dpto. de Puno, Perú \\
\hline & 10937 & Hacienda Checayani, Dpto. de Puno, Perú \\
\hline & 12017 & Sin procedencia \\
\hline \multirow{5}{*}{ Telmatobius mayoloi } & 20470, 20471, 20472, 20473, 20474, 20550 (larvas) & Conococha, Dpto. de Ancash, Perú \\
\hline & 7417 (larvas), 7422 (larvas) & Rio Santa, Dpto. de Ancash, Perú \\
\hline & 20489 (larvas) & Pachacoto, Dpto. de Ancash, Perú \\
\hline & 20479,20480 & Aguascocha, Ancash \\
\hline & $20478,20486,20488$ & Catac-Ancash \\
\hline \multirow{3}{*}{ Telmatobius peruvianus } & $19604,19605,19606,19607,19608,19609$ & Caplina, Dpto. de Tacna, Perú \\
\hline & 21343 (larvas) & Palca, Dpto. de Tacna, Perú \\
\hline & 12418 & Torata, Dpto. de Tacna, Perú \\
\hline \multirow{5}{*}{ Telmatobius rimac } & 12817 & Marcahuasi, Dpto. de Lima, Perú \\
\hline & 19229 (larvas), 12458, 12459, 12460, 12629 & Canta, Dpto. de Lima, Perú \\
\hline & 20552 & Coris, Dpto. de Ancash, Perú \\
\hline & $12489,12495,12509,12552$ & Ocros, Dpto. de Ancash, Perú \\
\hline & $10330,10334,10337,12712,12713$. & Sin procedencia \\
\hline \multirow{2}{*}{ Telmatobius truebae } & $\begin{array}{l}6183,6184,6185,12364,12365,12366,12367,12368,12369, \\
12370\end{array}$ & Bongara, Dpto. de Amazonas, Perú \\
\hline & 19552 (larvas), 22678 (larvas) & Leimebamba, Dpto. de Amazonas, Perú \\
\hline
\end{tabular}

